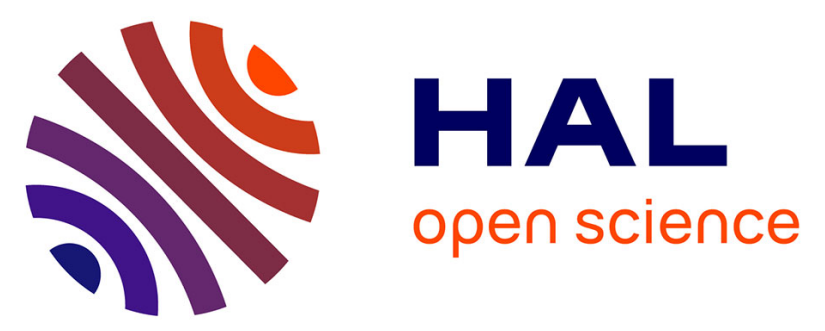

\title{
Dispositif expérimental permettant de mesurer simultanément l'absorption hydrique et la transpiration d'une jeune plante cultivée sur sol. - Premiers résultats obtenus
}

Jean Morizet, Robert Falcimagne, Michel Martignac

\section{To cite this version:}

Jean Morizet, Robert Falcimagne, Michel Martignac. Dispositif expérimental permettant de mesurer simultanément l'absorption hydrique et la transpiration d'une jeune plante cultivée sur sol. - Premiers résultats obtenus. Agronomie, 1987, 7 (7), pp.541-546. hal-00885025

\section{HAL Id: hal-00885025 https://hal.science/hal-00885025}

Submitted on 1 Jan 1987

HAL is a multi-disciplinary open access archive for the deposit and dissemination of scientific research documents, whether they are published or not. The documents may come from teaching and research institutions in France or abroad, or from public or private research centers.
L'archive ouverte pluridisciplinaire HAL, est destinée au dépôt et à la diffusion de documents scientifiques de niveau recherche, publiés ou non, émanant des établissements d'enseignement et de recherche français ou étrangers, des laboratoires publics ou privés. 


\title{
Dispositif expérimental permettant de mesurer simultanément l'absorption hydrique et la trans- piration d'une jeune plante cultivée sur sol. Premiers résultats obtenus
}

\author{
Jean MORIZET, Robert FALCIMAGNE \& Michel MARTIGNAC \\ I.N.R.A., Station d'Agronomie, 12, avenue du Brézet. Centre de Recherches de Clermont-Ferrand-Theix, \\ F 63039 Clermont-Ferrand
}

RÉSUMÉ Cette note présente un dispositif expérimental permettant de mesurer simultanément l'absorption hydrique et la
transpiration d'une jeune plante cultivée sur sol. Les parties aériennes sont placées dans une chambre à circuit
ouvert et la transpiration est calculée à partir du débit d'air qui traverse l'enceinte et des teneurs en vapeur d'eau
mesurées à l'entrée et à la sortie de celle-ci. L'absorption hydrique est déterminée par pesée du compartiment
racinaire après avoir mis à l'air libre la partie supérieure des racines afin de réduire la liaison mécanique entre
milieu racinaire et partie aérienne de la plante. La précision des mesures est discutée et quelques résultats sont
décrits, concernant l'évolution de la transpiration, de l'absorption hydrique et du bilan hydrique, au cours d'un
cycle desséchement-réhydratation observé sur 3 journées consécutives.

Mots clés additionnels : Bilan hydrique, potentiel hydrique. and transpiration of a young plant grown on soil.

This note presents a experimental device for measuring simultaneously water absorption and transpiration rates of a young plant grown in soil. The shoot was installed in a transpiration chamber. The transpiration rate was monitored by gaseous exchange measurements as usually done in open-circuit systems. The water absorption rate was determined by weighing the root compartment. For this purpose, the mechanical link between root compartment and shoot had to be reduced. This was achieved by removing the soil particles around the upper roots. Some examples of the time course of transpiration-absorption rates and of water balance are given, for a 3-day drought-rewatering cycle. The accuracy of the data is discussed.

Additional key words : Water balance, water potential.

\section{INTRODUCTION}

L'intégration dans le temps du bilan en eau d'une plante détermine son état hydrique global à chaque instant. Suivant les conditions édaphiques et atmosphériques qui règlent respectivement l'offre et la demande, 2 possibilités se présentent :

- soit les débits d'absorption et de transpiration sont égaux et la plante maintient son état hydrique au niveau antérieur, grâce à un régime de flux conservatif,
- soit les débits sont différents et le régime de flux non conservatif aboutit à une modification progressive de l'hydratation des tissus végétaux.

Les mesures simultanées de l'absorption et de la transpiration apparaissent donc comme un moyen privilégié pour suivre l'évolution de l'état hydrique d'une plante à travers les fluctuations des facteurs du milieu. Par ailleurs, ces informations peuvent être utilement complétées par l'évaluation de l'eau de croissance qui représente habituellement une fraction faible mais non négligeable de l'eau absorbée. 
Un autre intérêt de ces mesures réside dans la possibilité de calculer la résistance au transfert de l'eau en phase liquide à partir des relations existant entre flux et potentiels hydriques aux niveaux racinaire et foliaire (KATERJI et al., 1983).

En culture hydroponique, les techniques pour déterminer simultanément l'absorption et la transpiration sont déjà connues et donnent satisfaction (MINGEAU, 1969 ; MORIZET, 1976 ; LASCEVE \& COUCHAT, 1980). Par contre, on se heurte à plus de difficultés pour apprécier l'absorption hydrique en culture sur sol. A notre connaissance, une seule technique a été proposée jusqu'ici pour mesurer l'absorption hydrique de plantes cultivées sur sol ; celle décrite par KRAMER en 1937. Le sol est humidifié par un cône poreux relié à un réservoir d'eau. Les variations de volume d'eau dans ce réservoir fournissent les quantités d'eau absorbées, aux changements près de teneurs en eau du sol. SLAVIK (1974) présente une revue bibliographique des potomètres du sol. Les auteurs cités : LEBEDEV \& SOLOLEv (1964), LeBedev et al. (1964) ont utilisé des appareils dont le principe de mesure est le même que celui de Kramer (1937), BraUn \& SCHMIDT (1972) ont estimé l'absorption de l'eau à partir des variations de niveau du plan d'eau, technique présentant là encore les inconvénients dus aux variations d'humidité du sol.

L'objectif de cette note est de présenter un nouveau dispositif expérimental permettant de suivre de façon continue la transpiration et l'absorption hydrique d'une jeune plante cultivée sur sol. Quelques résultats obtenus seront ensuite décrits.

\section{DESCRIPTION DU DISPOSITIF EXPÉRIMENTAL}

Le dispositif expérimental comprend une chambre de transpiration et un montage destiné à mesurer l'absorption hydrique (fig. 1). Nous décrirons brièvement le premier qui est d'utilisation courante et nous présenterons plus en détail le second qui fait l'objet principal de cette note.

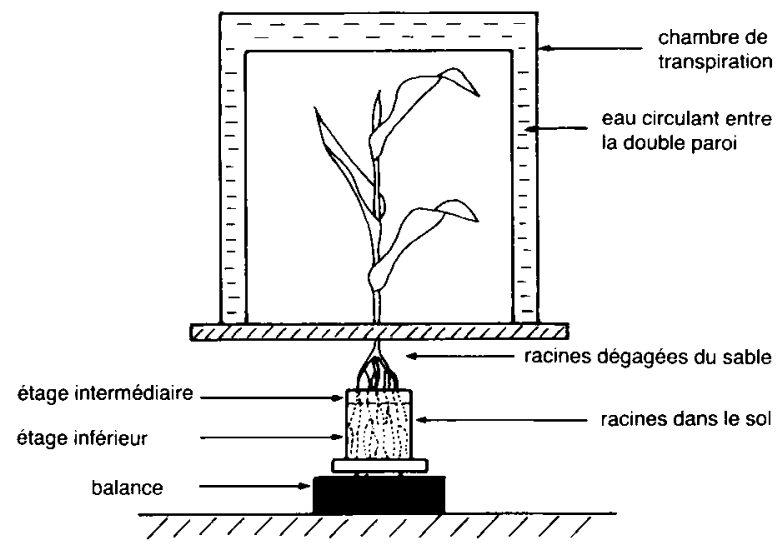

Figure 1

Schéma du dispositif expérimental pour mesurer l'absorption hydrique en condition de culture sur sol.

Sheme of experimental device for measuring the water absorption of a young plant grown on soil.

\section{A. Chambre de transpiration}

La partie aérienne de la plante est placée dans une enceinte à circuit ouvert. Celle-ci a un volume intérieur de 85 litres et possède une double paroi en plexiglass dans laquelle circule de l'eau maintenue à $20^{\circ} \mathrm{C}$ $\pm 0,5^{\circ} \mathrm{C}$. Le jour, l'éclairage est assuré par des lampes OSRAM HQITS délivrant une énergie lumineuse (P.A.R.) de 90 watts $\mathrm{m}^{-2}$ pendant une photopériode de $12 \mathrm{~h}$. La régulation hygrométrique est gérée par ordinateur type MACSYM (ANALOG DEVICES) qui commande l'ouverture d'une électrovanne 3 voies, calculée à l'aide d'une boucle proportionnelle intégrée à partir des données fournies par la sonde hygrométrique de sortie. L'électrovanne assure le mélange de l'air sec fourni par un compresseur et de l'air humidifié par passage dans un caisson d'humidification.

La transpiration est calculée à partir du débit d'air traversant la chambre et mesuré par un débitmètre massique type FC 262 (TYLAN) et à partir des températures de point de rosée déterminées à l'entrée et à la sortie de l'enceinte par des transmetteurs de point de rosée type DEW-10 (GENERAL EASTERN).

\section{B. Mesure de l'absorption hydrique}

Celle-ci est effectuée par pesée du milieu racinaire, après avoir dégagé la partie supérieure des racines. $\mathrm{Si}$ ces dernières sont suffisamment souples, on réduit ainsi la liaison mécanique entre milieu racinaire et partie aérienne de la plante et les variations de poids enregistrées correspondent à l'absorption.

Nous décrirons successivement le dispositif de culture conçu pour faciliter le dégagement des parties supérieures des racines, ensuite la procédure proprement dite de mesure de l'absorption.

\section{Dispositif de culture (fig. 2)}

La germination de 3 graines est effectuée dans un étage supérieur cylindrique, contenant du sable quartzeux de BIOT dont la granulométrie est comprise entre 1,6 et $3 \mathrm{~mm}$. Le sable est retenu par une toile métallique à mailles fines, placée à la partie inférieure de

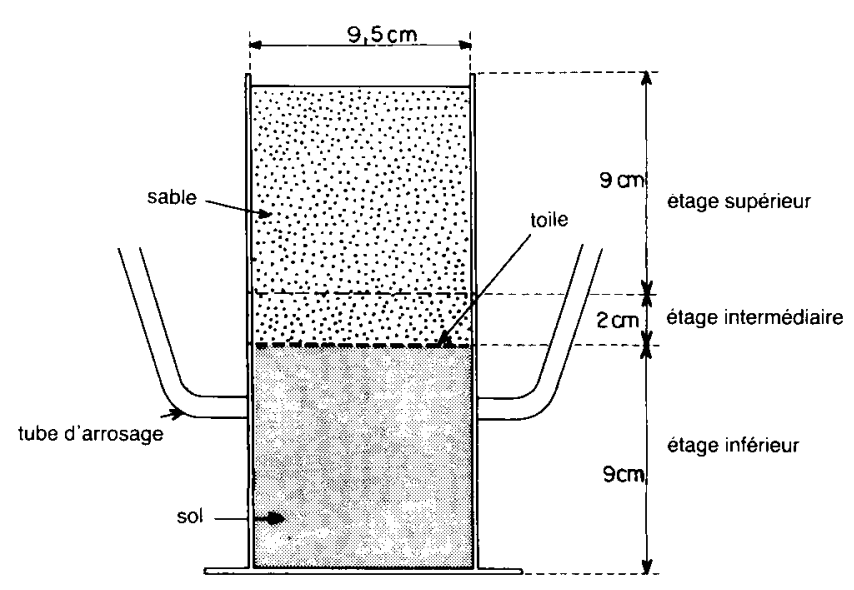

Figure 2

Dispositif expérimental pour la culture sur sol.

Experimental device for a plant grown on soil. 
l'étage intermédiaire (fig. 2). L'arrosage est assuré avec de la solution nutritive $T_{1}$ contenant tous les éléments minéraux indispensables à la croissance de la plante (MORIZET, 1976). Une seule plantule est ensuite laissée par récipient. Quand les racines apparaissent entre les mailles de la toile, c'est-à-dire une dizaine de jours après le semis, l'ensemble des 2 étages supérieur et intermédiaire est disposé sur l'étage inférieur. L'assemblage entre les étages est assuré par une bande de ruban adhésif. L'étage inférieur contient un sol humifère sableux arrosé régulièrement jusqu'à la capacité de rétention (humidité pondérale $=40$ p. 100) par 2 tuyaux disposés latéralement.

Les mesures sont effectuées sur des plantes de tournesol ou de maïs âgées de 4 à 5 semaines de façon que leur taille soit compatible avec le volume de l'enceinte expérimentale.

\section{Mesure proprement dite de l'absorption}

La mise en place de la plante dans le dispositif expérimental est effectuée de la façon suivante. Le cylindre supérieur est retiré. Le sable contenu dans celui-ci est ainsi libéré et les racines sont délicatement dégagées à l'aide d'un pinceau jusqu'au niveau supérieur de l'étage intermédiaire. Le sable est maintenu en place dans ce dernier pour éviter l'évaporation directe à partir du sol. Un tuteur est placé temporairement afin de maintenir la partie aérienne de la plante et permettre l'introduction de celle-ci dans la chambre de transpiration. Le compartiment racinaire est alors posé sur le plateau d'une balance à sortie analogique, dotée d'un système de compensation électromagnétique qui maintient le plateau immobile (METTLER PE 3600) (fig. 1). Le tuteur est ensuite retiré.

Ainsi disposés, les segments supérieurs des racines qui sont à l'air libre, peuvent être soumis à une certaine tension qui fausse la pesée. Pour éviter cet inconvénient, il convient de relâcher la contrainte en relevant légèrement la position du support de la balance.

Afin de vérifier que la liaison racinaire est suffisamment souple pour ne pas perturber la pesée, nous avons ajouté à la pipette $5 \mathrm{ml}$ d'eau sur le compartiment racinaire et nous avons noté la variation de poids qui en résultait sur la balance. Sur 10 répétitions effectuées sur 5 plantes différentes, nous avons obtenu une augmentation de poids moyenne de 4,98 g avec un intervalle de confiance de $\pm 0,05 \mathrm{~g}$ $(P=0,05)$.

Des saisies de données sont effectuées toutes les 3 minutes par l'ordinateur qui calcule la perte de poids pendant ce laps de temps et en déduit l'absorption hydrique par minute.

Les courbes de transpiration et l'absorption sont tracées simultanément sur enregistreur graphique multivoies (LINSEIS, 2065).

\section{Précision des mesures}

\section{Transpiration}

La transpiration $\mathrm{T}$ est fournie par la relation suivante :

$$
\mathrm{T}=\mathrm{D} \cdot(\mathrm{Cs}-\mathrm{Ce})
$$

avec $\mathrm{D}=$ débit d'air qui traverse la chambre

Cs = concentration en vapeur d'eau dans la chambre et à la sortie de la chambre

$\mathrm{Ce}=$ concentration en vapeur d'eau à l'entrée de la chambre.

L'erreur relative commise sur la transpiration est donc :

$$
\Delta \mathrm{T} / \mathrm{T}=\Delta \mathrm{D} / \mathrm{D}+(\Delta \mathrm{Cs}+\Delta \mathrm{Ce}) /(\mathrm{Cs}-\mathrm{Ce})
$$

La précision du débitmètre, fournie par le constructeur, est de 2 p. 100 . Comme d'autre part, la concentration en vapeur d'eau $C$, à la température ambiante de $21^{\circ} \mathrm{C}$, est fonction de la température du point de rosée $\theta$, selon la relation :

$$
\mathrm{C}=4,5 \cdot 10^{[7.5 \cdot \theta /(237,3+\theta)]},
$$

on en déduit :

$$
\mathrm{dC}=18440 \cdot 10^{[7,5 \cdot \theta /(237,3+\theta)]} /(237,3+\theta)^{2} \mathrm{~d} \theta
$$

En admettant une erreur de $0,1{ }^{\circ} \mathrm{C}$ dans les données fournies par les hygromètres à point de rosée (les écarts entre les 2 appareils placés dans la même ambiance, ne sont jamais supérieurs à cette valeur), on peut écrire :

$$
\Delta \mathrm{C}=1844 \cdot 10^{[7,5 \cdot \theta /(237,3+\theta)]} /(237,3+\theta)^{2}
$$

La relation (5) permet d'expliciter (2) :

$$
\begin{aligned}
\Delta \mathrm{T} / \mathrm{T}=\Delta \mathrm{D} / \mathrm{D} & +410\left[\mathrm{Ms} /(237,3+\theta \mathrm{s})^{2}+\right. \\
& \left.+\mathrm{Me} /(237,3+\theta \mathrm{e})^{2}\right] /(\mathrm{Ms}-\mathrm{Me})
\end{aligned}
$$

avec :

$$
\begin{aligned}
& \mathrm{Ms}=10^{7,5 \theta s /(237,3+\theta \mathrm{s})} \\
& \mathrm{Me}=10^{7,5 \theta \mathrm{c} /(237,3+\theta \mathrm{c})}
\end{aligned}
$$

La précision des mesures de transpiration est donc fonction de $\theta$ s et $\theta \mathrm{e}$ : températures du point de rosée respectivement à la sortie et à l'entrée de la chambre.

La figure 3 donne l'erreur maximale commise sur la transpiration en fonction de l'hygrométrie de l'air d'entrée et pour 3 hygrométries à l'intérieur et à la

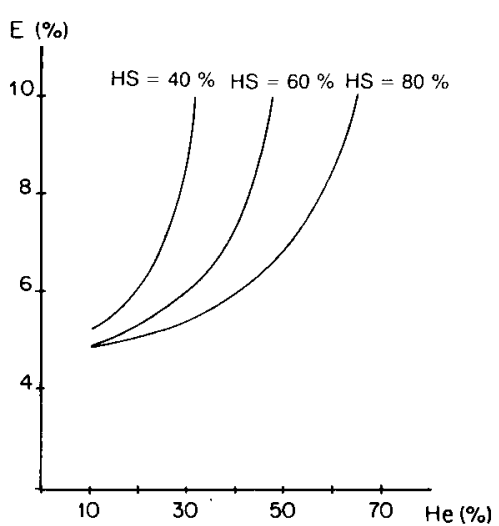

Figure 3

Erreur maximale (E) commise sur la transpiration, en fonction de l'hygrométrie de l'air d'entrée (He) et pour 3 hygrométries de sortie (Hs).

Maximal error (E) on the transpiration, as a function of in-flow $R H$ (He) and 3 out-flow $R H$ values (Hs). 
sortie de l'enceinte : $40,60,80$ p. 100 . Pour être sûr d'avoir une erreur maximale inférieure à 10 p. 100 , il faut régler le débit d'air à l'intérieur de la chambre de sorte que l'écart entre hygrométries de sortie et d'entrée soit au moins de 10 à 15 p. 100 .

\section{Absorption}

La balance utilisée pour peser le compartiment racinaire est sensible au centigramme. Si l'on admet que les racines exposées à l'air sont suffisamment souples pour ne pas diminuer la précision de la pesée, la limite supérieure de l'erreur commise sur la différence de poids calculée à 3 minutes d'intervalle est $2 \times 10 \mathrm{mg}$ $=20 \mathrm{mg}$. L'erreur relative dépend de la différence de poids. Celle-ci, au cours de la phase lumineuse, a une valeur généralement comprise entre 200 et $400 \mathrm{mg}$, ce qui conduit respectivement à une précision de 10 p. 100 et 5 p. 100.

\section{Erreur estimée expérimentalement}

Pour la détermination expérimentale de l'erreur commise sur la transpiration, on a comparé les pertes de poids d'une cuvette remplie d'eau placée à l'intérieur de la chambre sur une balance enregistreuse et l'évaporation calculée à partir du débit d'air et des températures de point de rosée à l'entrée et à la sortie.

Sur $5 \mathrm{~h}$, les différences entre les 2 méthodes représentent $2,0 \mathrm{~g}$ sur une perte de poids de $94 \mathrm{~g}$, soit une erreur de 2 p. 100 commise sur l'évapotranspiration. Si l'on fait les comparaisons par quart d'heure, l'intervalle de confiance $(P=0,05)$ calculé sur les écarts est de $4,5 \mathrm{~g}$, ce qui correspond à une erreur de 5 p. 100.

Cette façon expérimentale de procéder fournit des valeurs plus optimistes sur la précision des mesures que les calculs théoriques développés auparavant. Ceci paraît logique, car ces derniers s'appliquent à des déterminations instantanées ou portant sur une période de très courte durée $(3 \mathrm{mn})$. Par contre, l'approche expérimentale fait une intégration sur une durée plus longue, ce qui a pour effet de diminuer l'erreur relative, si l'on admet, comme il est probable que les «erreurs instantanées » ne sont pas de nature systématique.

\section{UN EXEMPLE DE RÉSULTATS OBTENUS SUR UNE JEUNE PLANTE DE MAÏS}

Voici les résultats obtenus sur un maîs ayant une surface foliaire de $750 \mathrm{~cm}^{2}$. L'air balayant la chambre a une hygrométrie de $50 \pm 1$ p. 100 .

\section{A. Suivi de l'absorption et de la transpiration au cours d'un cycle de dessèchement-réhydratation}

Quand la disponibilité hydrique dans le sol n'est pas facteur limitant (fig. 4a), le régime de flux est conservatif pendant la presque totalité de la phase lumineuse. Ce n'est que pendant la $1^{\text {re }}$ heure qui suit l'allumage, que la transpiration excède nettement l'absorption ou que leurs oscillations sont décalées
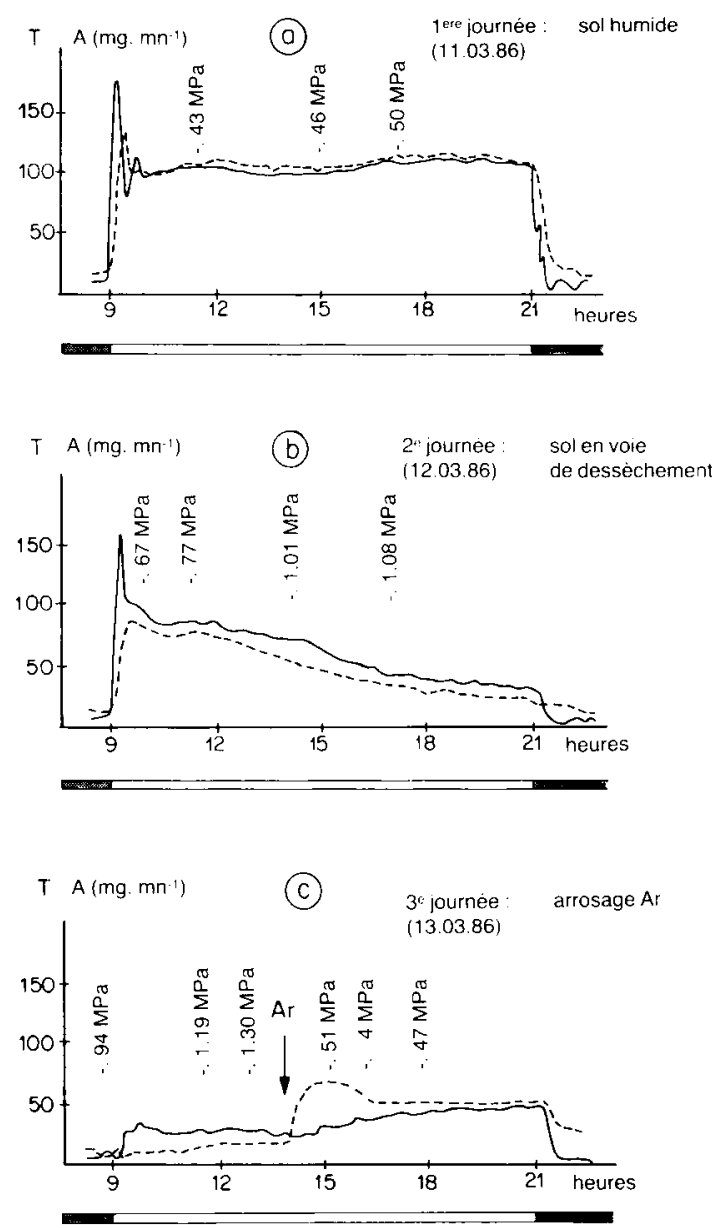

Figure 4

Evolution de la transpiration $(T:-$ ) et de l'absorption ( $A$ :--) d'une jeune plante de maïs, au cours de 3 journées consécutives. Les potentiels hydriques foliaires (MPa) sont notés au-dessus des courbes.

$\square$ : phase lumineuse Ar: Arrosage

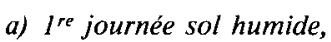

b) $2^{e}$ journée sol en voie de dessèchement,

c) ze journée arrosage.

Change in transpiration ( $T:-(-)$ and absorption ( $A$ : --) of a young maize plant during 3 consecutive days. The leaf water potentials $(M P a)$ are reported above the curves.

$\square$ : light period dark period Ar: Watering

a) first day moist soil,

b) second day drying soil,

c) third day watering.

dans le temps. Cependant, l'amplitude du pic transpiratoire initial après allumage, est probablement exagérée à cause du système de régulation hygrométrique qui modifie rapidement et d'une façon importante l'humidité de l'air d'entrée, quand il y a variation des conditions climatiques à l'intérieur de l'enceinte. Par la suite, le léger excédent de l'absorption de la transpiration, qui représente en moyenne $3,2 \mathrm{mg} \mathrm{min}^{-1}$, peut être attribué à l'eau de croissance.

La figure $4 \mathrm{~b}$ représente l'évolution du flux hydrique observé le lendemain, au cours du développement de la sécheresse. Les potentiels hydriques foliaires, mesurés par hygromètre à point de rosée L 51 (WESCOR) décroissent pour atteindre $-1,08 \mathrm{MPa}$ à $17 \mathrm{~h}$. 
L'absorption et la transpiration diminuent simultanément. Mais, contrairement à la journée précédente, le niveau de-l'absorption est inférieur à celui de la transpiration, ce qui traduit un bilan hydrique déficitaire à l'intérieur de la plante.

Enfin, au cours de la $3^{\mathrm{e}}$ journée (fig. 4c), le sol est ramené à la capacité de rétention. A la suite et très rapidement, le flux d'absorption augmente. Le potentiel hydrique foliaire remonte également, mais un peu moins vite. Enfin, la transpiration s'élève lentement, d'une façon linéaire.

\section{B. Evolution du bilan hydrique (fig. 5)}

Au cours de la $1^{\text {re }}$ journée, le bilan constamment positif, se traduit par un gain final de $7 \mathrm{~g}$ environ, observé en fin de nuit.

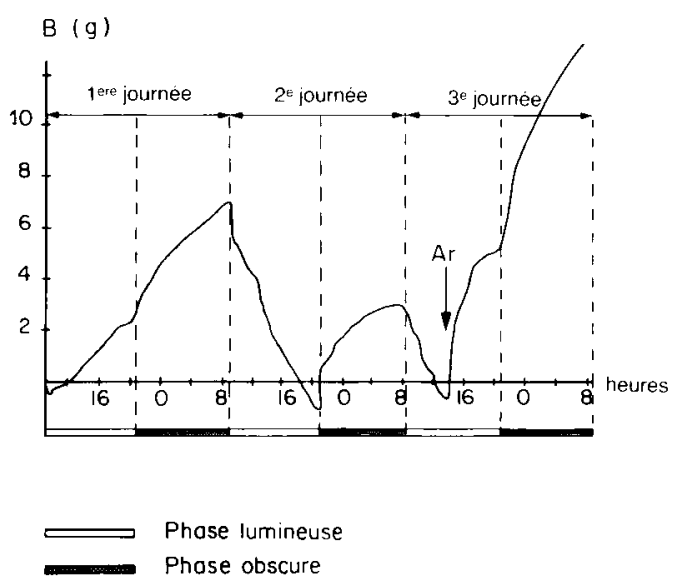

Figure 5

Evolution du bilan hydrique, au cours de 3 journées consécutives.

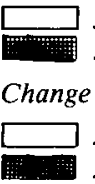

phase lumineuse phase obscure Ar : Arrosage in water balant period dark period

Puis la période lumineuse de la $2^{\mathrm{e}}$ journée se caractérise par une inversion de tendance corrélée à la décroissance des potentiels hydriques foliaires. A la fin de cette période, le bilan devient négatif par rapport à la référence de départ. La nuit suivante est marquée par une légère réhydratation malgré la sécheresse du sol.

Au début de la $3^{\mathrm{e}}$ journée, le bilan redevient négatif jusqu'à l'arrosage à 13 h 45 . Après celui-ci, la reprise hydrique est très rapide et s'accélère même en début de nuit.

\section{DISCUSSION ET CONCLUSION}

Le dispositif expérimental décrit dans cette note, permet de suivre, de façon continue, l'absorption hydrique et la transpiration par des méthodes totalement indépendantes et donc de déterminer à chaque instant le bilan hydrique de jeunes plantes cultivées sur sol.

La précision obtenue est satisfaisante. Pour l'absorption, cependant, il importe que les racines restent suffisamment souples pour ne pas fausser la pesée, condition généralement remplie avec des plantes jeunes.

L'absorption ainsi mesurée, représente plus précisément le flux hydrique qui traverse les racines selon un plan situé au niveau de la « dissociation mécanique » entre le compartiment racinaire placé sur le plateau de la balance et la partie supérieure des racines qui se trouve hors du sol. Ce plan se situe probablement juste au-dessus du sable de l'étage intermédiaire, là où les racines dégagées sont les plus fines et donc les plus souples. Il en résulte que le bilan hydrique calculé à partir des données mesurées de l'absorption et de la transpiration, concerne les parties aériennes et la fraction des racines dégagées mais non le chevelu racinaire contenu dans le sol sous-jacent.

Pour avoir le bilan hydrique réel, c'est-à-dire celui obtenu en tenant compte de la croissance de la plante, il conviendrait de défalquer "l'absorption de croissance » de l'absorption brute mesurée. Une approche en ce sens pourrait très certainement être tentée en mesurant simultanément l'assimilation nette, ce qui permettrait une évaluation de la matière sèche formée.

Les premiers résultats obtenus montrent qu'un régime de flux conservatif s'installe très rapidement après passage de la phase obscure à la phase lumineuse et qu'il se maintient durant toute la journée à condition que la disponibilité hydrique au sein du sol ne soit pas limitante. KRAMER (1937) avait observé des flux transpiratoires nettement supérieurs aux flux d'absorption au cours de la matinée et en début d'après-midi et une inversion de cette tendance en fin d'après-midi. L'auteur fournit lui-même une explication à ce phénomène qui serait lié, en partie du moins, à un artefact causé par le retard dans l'irrigation du sol. Il en résulterait un dessèchement de celui-ci et donc une sous-estimation de l'absorption dans la première partie de la journée. Le phénomène inverse se produirait en fin de journée.

Ce dispositif est susceptible de fournir des données intéressantes, concernant les relations entre potentiels hydriques foliaires et flux hydriques à travers la plante et sur les résistances en phase liquide qui en sont déduites. 


\section{RÉFÉRENCES BIBLIOGRAPHIQUES}

Braun H. J., Schmidt P., 1972. Methodische Versuche zur direkten Messung des absoluten Wasserverbrauches der Holzpflanzen. $Z$. Pflanzenphysiol., 66, 337-342.

Katerji N., Hallaire M., Perrier A., Durand R., 1983. Transfert hydrique dans le végétal. I. Modélisation à l'échelle du couvert végétal en conditions naturelles. Acta Oecologica, Oecol. Plant., 4, 11-26.

Kramer P. J., 1937. The relation between rate of transpiration and rate of absorption of water in plants. Am. J. Bot., 24, 10-15.

Lasceve G., Couchat Ph., 1980. Le transfert de l'eau dans la plante en régime transitoire. Ann. agron., 31, 273-283.

Lebedev G., Chuchkin V. G., Sabinina E. D., Bryukvin V. G. 1964. An apparatus for continuous recording of water uptake by plants. Fiziol. Rast., 2, 1110-1114 (en russe).
Lebedev G. V., Solovev A. K., 1964. An apparatus of studying the rate of water uptake by plants. Fiziol. Rast., 2, $752-756$ (en russe).

Mingeau M., 1969. Action de la nutrition minérale sur l'économie de l'eau dans la plante. I. Equilibre absorption-transpiration. Ann. agron., 1969, 20, 263-276.

Morizet J., 1976. Description d'un potomètre enregistreur, un exemple d'observation portant sur une journée. Ann. agron., 27, 247-257.

Slavik B., 1974. Methods of studying plant water relations. Ed. Academia Praha, p. 212. 